\title{
Simple Expert System for Intelligent Control and HCI for a Wheelchair fitted with Ultrasonic Sensors
}

\author{
David Sanders \\ School of Mechanical and Design Engineering \\ University of Portsmouth, Portsmouth, UK \\ david.sanders@port.ac.uk \\ Giles tewkesbury \\ School of Mechanical and Design Engineering \\ University of Portsmouth, Portsmouth, UK \\ giles.tewkesbury@port.ac.uk
}

Peter Omoarebun

School of Mechanical and Design Engineering University of Portsmouth, Portsmouth, UK peter.omoarebun1@myport.ac.uk

\author{
Malik Haddad \\ School of Mechanical and Design Engineering \\ University of Portsmouth, Portsmouth, UK \\ malik.haddad@port.ac.uk \\ Mohamad Thabet \\ School of Energy and Electronic Engineering \\ University of Portsmouth, Portsmouth, UK \\ mohamad.thabet@port.ac.uk \\ Tom Barker \\ Gems Sensors \\ Lennox Road, Basingstoke, RG22 4AW, UK \\ tom.barker@gems-sensors.co.uk
}

\begin{abstract}
Using an expert system to make driving a poweredwheelchair less problematic is investigated. The system interprets sensor and joystick signals and then mixes them and improves that collaboration to control speed and direction. Ultrasonic sensors are used to identify hazardous circumstances and suggest a safer direction and speed. Results from drivers completing a series of timed routes are presented. Users completed tests using joysticks to control their chair with and then without a microcomputer and sensor system. A recent system is used to compare and contrast the results. This new system consistently performed quicker than the recent system. It also appears that the quantity of support provided by the sensors and microcomputer should be adjusted depending on situations and surroundings
\end{abstract}

Keywords- Disabled; sensor; wheelchair; expert; ultra-sonic;

\section{INTRODUCTION}

A simple expert system [1,2] is described that controls a powered-wheelchair [3]. Ultrasonics [4] are used to identify dangerous circumstances and suggest safer directions and speeds. Systems used at the moment [5-8] are likely to rely greatly on vision and the experience of the drivers. This paper describes methods to make the driving task easier using an expert system to interpret sensor and joystick signals.

The way that the wheelchair interacts with a human operator might reduce effectiveness [5-10]. Improvements to the interaction are investigated. Algorithms mix data from the joystick controlling a wheelchair with data from the ultrasonics. In the past, the control systems have often been open-loop. Drivers indicate a bearing and the wheelchair moves on that bearing. Disturbance can be introduced because of differences in the wheels or the reaction of the wheels and chair to slopes, hills and surfaces [11]. Drivers react to those disturbances and then revise their desired trajectory. The intelligent system described here needed a control algorithm and sensors. The algorithm was to process data from sensors and driver's joysticks and use the information generated to assist the drivers.

Powered-wheelchairs have often been guided with a joystick [5-13] although there are other devices: switches [14], pointers $[15,16]$ or virtual reality transducers [17]. And poweredwheelchairs need to avoid objects, wheelchairs, vehicles and/or people [18,19]. Various local sensors have been used with wheelchairs: light or laser [20], ultrasonics [5-13] or infra-red [21]. Positioning has used tilt, gyro, odometry or acoustic [22]. GPS [23] does not work well indoors or in shielded areas but assisted GPS [24] may be useful. Vision [25-31] needs data processing but as cost has reduced and computing power increased [32] they are more popular. Sensors are becoming more capable but human drivers are still the best data source. Ultrasonics were selected as they are simple, cost effective and robust [5-12, 33-35]

\section{WHEELCHAIR SYSTEMS}

A powered-wheelchair with two large driving wheels and two trailing casters was used. Cameras were fixed at the front of the chair in between the two large wheels. Three pairs of ultrasonic transducers were fixed to the wheelchair. Two were fixed above each large driving wheel and a third was fixed at the front in the center. Receiver / transmitter pairs were fixed to the front of the chair $[3,9]$ and they transmitted $1 \mathrm{~ms}$ sound pulses. The pulse reflected back from objects and range was determined using the time it took for a pulse to get back to the receiver. The 
simple sound image could then be converted into a depiction of the surroundings. Obstacles ahead of the chair were sensed.

Links between joysticks and wheelchairs were disconnected. Instead, a microcomputer handled control in 3 possible modes:

- The microcomputer interrogated the ultrasonics and modified the bearing taken by the chair using the:

- New algorithms

- Recently published algorithms.

- Joystick signals drove the wheelchairs directly.

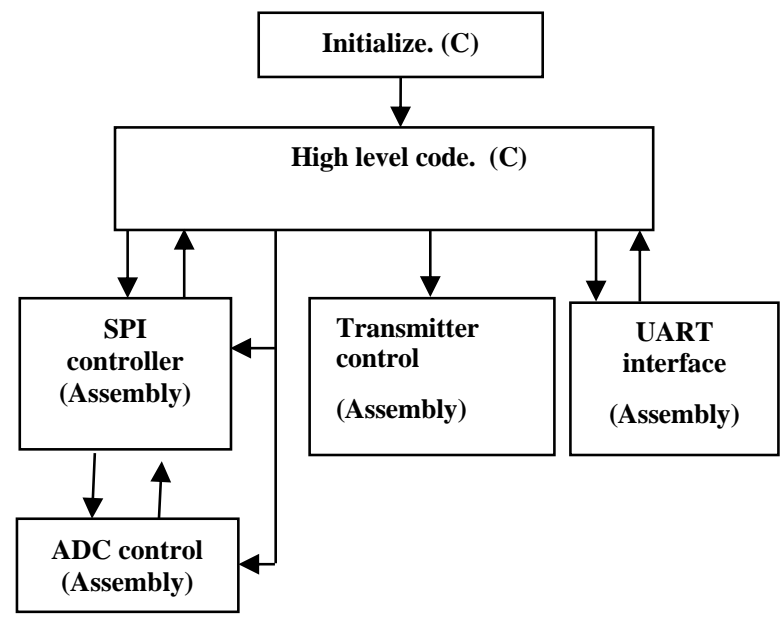

Figure 1. Code structure showing Assembly and C Modules.

Fig. 1 shows the hierarchical code structure. Algorithms applied these rules: Movements were controlled and smooth; Users remained in control overall; Trajectories were only modified when needed. Data from the sensors was used to generate an imaginary potential field around obstacles [36].

Transmitters needed a $3 \mathrm{~ms}$ pulse to reach maximum power output. Longer pulses of sound contained more energy so that the pulses could detect obstacles at a longer range. Sound travels at about $330 \mathrm{~m} / \mathrm{s}$. If the pulse of sound lasts for three milliseconds then pulse length is approximately one meter. Minimum range was 0.5 meters for that pulse length. As a shorter range was needed, a shorter pulse was required. Lengths of $1 \mathrm{~ms}, 500$ microseconds, 100 microseconds and 50 microseconds were investigated. The lengths of the pulses could be automatically switched by a "range finder" depending on ranges to obstacles. If nothing was detected, the "range finder" searched by slowly incrementing the length of the sound pulse so that the range was increased.

Despite the advantages, ultrasonics was relatively noisy and often gave misreadings. Misreadings were filtered using Histogramic In-Motion Mapping. Volumes ahead of the sensors created a straightforward lattice was stored as an array consisting of 3 volumes: immediate, nearby and distant. When something was detected then the range to the object was classified: immediate, nearby and distant. Three ultrasonic transducers were fixed to the chair so that their ultrasonic beams covered the volumes ahead of the chairs. Three sensors are shown in Fig. 2.
The elements of the grid occupied by an object were incremented by a relatively high number, e.g.: 4. Other elements were reduced by a lower number, e.g.: 1. Elements within the arrays had maximum values of 15 and minimum values of 0 . Objects detected within the volume represented by a grid element caused that element of the grid to increase in value. Arbitrary readings in any other grid elements briefly increased them, but false readings were reduced when the systems updated. When an obstacle moved into a different grid element then the new grid element increased in value and the old grid element reduced. Reliable ranges were obtained within $0.5 \mathrm{~s}$. The structure of the object detection process is shown in Fig. 3.

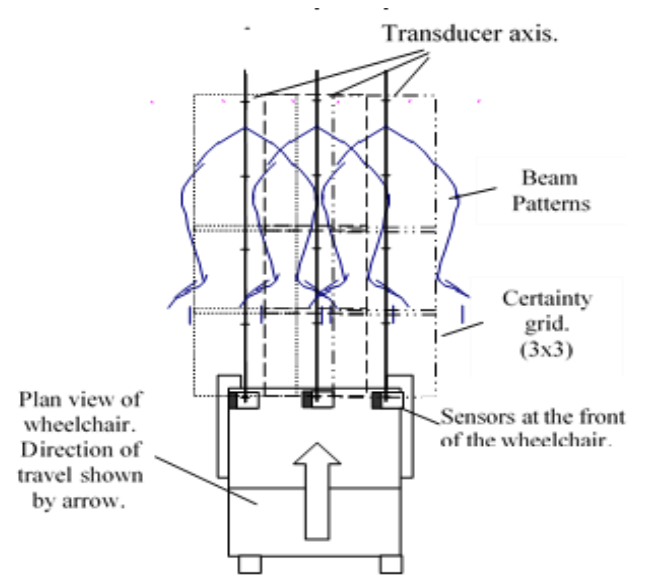

Figure 2. Three sensors and the array representing the volume ahead

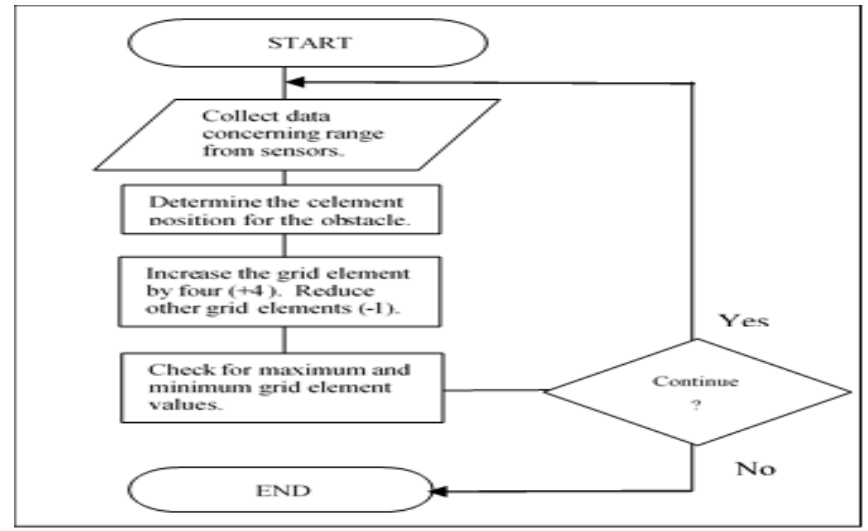

Figure 3. Structure of the histogrammic object detection process

\section{ALGORITHMS TO INTERPRET THE JOYSTICK}

The joystick fitted to the wheelchairs was a Penny \& Giles Joystick. It contained $2 \mathrm{x}$ potentiometers that provided two voltages that represented the joystick position that were read by an ADC that converted them to Cartesian co-ordinates. They were converted into a more convenient polar co-ordinate form: $|\mathbf{J}| \angle \theta$. Where $\angle \theta$ was the joystick angle representing the desired bearing and $|\mathbf{J}|$ was the desired speed. The mathematics functions provided with the $\mathrm{C}$ libraries were used, for example with square root and arctan for converting to polar coordinates.

Joystick angle was digitized and desired direction was estimated so that algorithms could measure how long a joystick was held in a position (representing desired direction and speed). 
Joystick magnitude and angle were calculated using:

Argument $=$ JS0/JS1; opposite $/$ adjacent $($ ATAN $)$

Bearing $=\operatorname{atan}(\operatorname{argument})$; Angle of joystick in radians

Magnitude $=\operatorname{sqrt}((\mathrm{JS} 1 * \mathrm{JS} 1)+(\mathrm{JS} 0 * \mathrm{JS} 0))$

Where JS1 and JS0 are Cartesian co-ordinates.

Angle and Magnitude were used to calculate the sector occupied by the joystick. Confidence and position was represented in an array, where each sector contained 2 array values.

"Angle Confidence" (0 to 15$)$ represented confidence of a joystick being within a sector for desired direction. "Magnitude" represented joystick position for desired speed.

Histogrammic representation acted as a pseudo-integrator so that if a joystick was held still then the grid element for that position was increased. Other grid elements were then reduced. The highest value grid element was used as the most confident position reading for the joystick. If a joystick occupied a grid element then that grid element quickly ramped up. Shaking hands on the joystick increased other grid elements for a moment but they then reduced every time the system updated. If the joystick was moved to a new grid element then the new grid element increased and the previous grid element reduced.

The position of the joystick was represented by a histogram. The highest histogram element represented desired direction. An example histogram for the joystick is shown in Fig. 4. A module called JSArray tested the angle and position of the joystick and decided which sector was occupied by the joystick. The corresponding element "angle confidence" (Aconf) was increased by 50. All other Aconf elements were then reduced by 25 . The occupied element increased and all other elements decreased. So histogram elements decayed quickly and increased relatively slowly. The weightings for decay and increasing were established experimentally. Wheelchairs were driven with various weighting factors.

\section{EXPERT SYSTEM}

Human rehabilitation engineers and expert drivers provided expert knowledge [37]. That knowledge [38-44] was intuitive for many people.

Systems needed to work in real-time [45-50] to assist the wheelchair drivers. The $2 \mathrm{x}$ real-time inputs were the joystick and the sensor system. The driver provided direction and speed and sensors collected data about their surroundings. A module named "Sensor-Expert" evaluated data from the sensors and if necessary recommended a new trajectory that would prevent a collision. Sometimes the data disagreed. Then an expert named "Fuzzy-Mixer" took into account both inputs and calculated the outputs to the motors. "Joystick Monitor" interpreted what the driver wanted the chair to do.

The system consisted of: "Doorway", "Sensor-Expert", "Joystick Monitor", and "Fuzzy-Mixer". "Fuzzy-Mixer" distributed control effort between sensors and the joystick. It matched recommendations from the sensors and joystick. It considered conflict between the two and obtained advice (information) from "Doorway", "Joystick Monitor" and "Sensor-Expert". "Proximity-Stop" was a failsafe function that halted the chair. "Fuzzy-Mixer" could override other inputs with "Proximity-Stop". "Fuzzy-Mixer" mixed sensor data and joystick confidence values and used them. If confidence was low then that meant that the system needed to avoid something $[46,47]$. If confidence in the joystick was high then that meant that the joystick position accurately reflected the wishes of the driver and then the sensors had less influence.

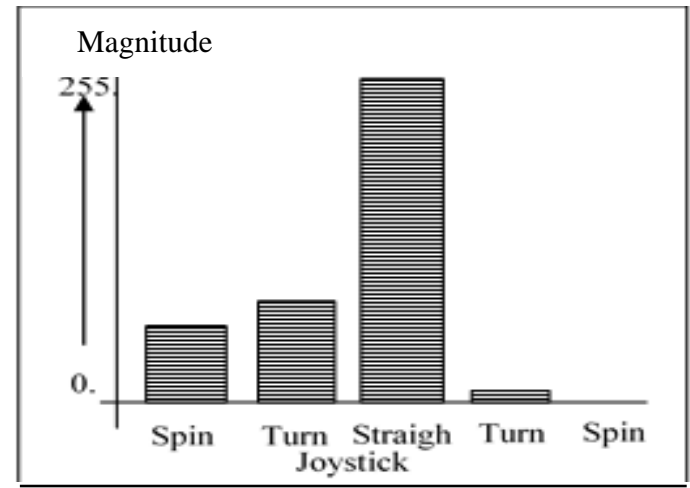

Figure 4. Representation of joystick using histograms

"Joystick Monitor" checked for changes in joystick position and consistency. A steady joystick position confirmed the desire of the driver. If a joystick moved more randomly then that suggested an out of control or unsure driver. Then the systems relied more on the sensor system to steer the wheelchair. "Sensor-Expert" applied sensor knowledge. "Sensor-Expert" constructed a grid for the sensor data and then recommended possible courses of action to steer the chair away from obstacles and avoid collision. "Sensor-Expert" did not consider the driver. "Doorway" was an object avoidance program that used information from data provided by "Sensor-Expert". "Doorway" was allowed to function or was over-ridden by "Fuzzy-Mixer". Sensor data merged with Joystick data so that:

$$
\begin{aligned}
& \text { Out }(\text { left })=\text { In (left) }- \text { F(right }) \\
& \text { Out (right) }=\text { In(right) - F(left) }
\end{aligned}
$$

Where output Out was the voltage to the controller, Input In was the voltage provided by the joystick, and F was distance to the closest object. They were vector quantities. There were two values, one for each wheel (right and left).

"Doorway" turned the chair away from the nearest obstacle, smoothly slowed the chair as the chair got close to obstacles and centered the chair between obstacles (for example a door frame). "Fuzzy-Mixer" distributed control to sensors or to the joystick depending on the situation and the environmental and the wishes of the driver. Relationships might be: (1) No sensors, All joystick, (2) No Joystick, All sensors, or (3) something between them. "Fuzzy-Mixer" assessed the inputs and the algorithms distributed control between the inputs:

LeftTarget $=(((\mathrm{JS} 0 *$ Aconf $[$ SectorJoystick $])+(($ LeftTarget$125) *(255$-Aconf $[$ SectorJoystick $]))) / 255)+125$;

RightTarget $=(((\mathrm{JS} 1 *$ Aconf $[$ SectorJoystick] $)+(($ RightTarget $-125) *(255-$ Aconf $[$ SectroJoystick] $))) / 255)+125$; 
Where LeftTarget and RightTarget are desired voltages.

$$
\begin{aligned}
& \text { JS0 and JS1 = Joystick values. } \\
& \text { Aconf[] = Joystick confidence. }
\end{aligned}
$$

Algorithms used distance functions to create target values for right and left voltages. Distance functions were:

$$
\begin{aligned}
& \text { LeftTarget }=2.5 * \text { result }[1]+110 ; \\
& \text { RightTarget }=2.5 * \text { result }[0]+110 ;
\end{aligned}
$$

Where: result[] = instantaneous range.

Sensor data were converted to a form that was compatible with Target (ADC) data. To make an assessment of the wishes of the user, the position of the joystick was needed. To achieve this, a joystick map was divided into sectors: Left Spin, Right Spin, Left Turn, Right Turn, Forward, Reverse and Stop. Sensor information was used to construct a Sensor-Byte. A rule set within "Sensor-Expert" was extracted from the mapping. A two to eight bit Sensor-Byte was created from the sensor arrays. Each sensor array had two bits to represent the position (or not) of an object in the array. Each pair of bits was expressed as 1 to 3 ( 2 bit binary code):

\section{0 nothing detected, \\ 1 object detected within "immediate" element, \\ 2 object detected within "nearby" element, \\ 3 object detected within "distant" element.}

These were used to search Sensor-Byte for object configurations so that "Sensor-Expert" could recommend action. "Sensor-Expert" recognized patterns in Sensor-Byte.

The software for the system was downloaded to a microcomputer mounted on the powered-wheelchair and tested by driving the powered-wheelchair in various environments.

A simplified Blackboard framework was used for the structure of the programs, similar to Hearsay II Blackboard78. The main module linked with a single module called "MainCode" and data was passed onto the blackboard. Software was constructed using a mix of low level language and high level language. The whole code was compiled into a machine-level file and loaded into on board non-volatile memory.

Algorithms were fast and predictable. If "Joystick" and "Sensor-Expert" were signifying "forward", a straight-forward bearing was set. Sensors were still interrogated to determine distance to objects. Speed was reduced as the wheelchair came close to objects. If the joystick was asking for a "Turn" then other algorithms were used. As an example one of the algorithms prevented a chair from driving fast into something in a right turn maneuver.

\section{TESTS}

Tests were conducted by driving the wheelchairs through various environments. System response was satisfactory so that the wheelchair could safely drive down a corridor and then align with the centre of a doorway when the joystick was held still in a pushed-forward position. The path of the wheelchair indicated that "Sensor-Expert" recommended satisfactory changes to bearings and speeds. Algorithms were effective in avoiding obstacles.

Drivers often used big joystick movements and the dynamics of the controller and the wheelchairs allowed that. Smaller movements led to a more sluggish reaction or were ignored. Bigger changes to controller inputs led to smoothed changes to trajectories.

Human drivers are capable and sophisticated. It was not the intention to replace the human driver but to assist them. The sensor assisted the drivers with steering their wheelchairs. The new system was initially tested in a laboratory before being tested in a variety of progressively more complicated environments using more complicated routes.

Drivers swiftly learnt how the wheelchairs worked, learnt to apply their controller (joystick) signals earlier and learnt to approximate stopping distances. Sets of test runs were undertaken to evaluate the speed of human drivers driving without any help and human drivers being assisted, using firstly an existing system [3,9] and then human drivers using the systems that have been described here. Time to complete routes was logged when operated by:

- Human driving alone (without any help).

- Drivers assisted by the existing previous systems.

- Drivers assisted by the systems described in this paper.

A driver guided their powered-wheelchair with the assistance of the sensor system. Researchers with digital laboratory clocks recorded times. Other researchers followed the powered-wheelchairs with stop watches. Tests were to:

- Capture comments and suggestions for improvement.

- Observe operation under joint human and computer control.

- Measure any improvement.

- Measure time taken by human drivers driving by themselves and measure time when driving with the assistance of expert systems and sensors.

Tests took place without sensors or assistance. Then tests were repeated with sensors engaged and then with assistance. For each test, obstacle courses were set up and poweredwheelchair drivers had to deal with the following environments:

LABORATORY: Objects on floors bounded by vertical walls.

EMPTY CORRIDOR: Sloping and flat surfaces; Vertical walls and doorways; Obstacles placed in a staggered arrangement.

COMPLICATED CORRIDOR: Sloping and flat surfaces; Doorways and vertical walls. Some items on the walls (for example door surrounds and radiators); Obstacles offset in a staggered formation and doorways to pass through;.

ENVIRONMENTS OUTSIDE: Complex environment with different sloping and flat surfaces; Bounded by different sloping and vertical edges; People walking around and through the environment; Various objects in the environment. 
Drivers were human and their performance varied so where possible, tests were conducted more than once. For each test series, drivers could repeat testing (with or without assistance) as often as they wished. That meant they could learn how systems behaved and then perform at their best.

Testing was considered to be fun and was well-liked. Tests encouraged competition and drivers wanted to beat others and beat their best. Sometimes drivers only completed the human driving test without assistance or only managed to complete the test with the assistance of the computer and sensors. Those results were discarded as comparisons could not be made for drivers.

Tests compared the speed of human drivers with drivers driving with assistance in typical environments and situations. If a fastest time was recorded by a driver in a set of tests then the driver made at least one attempt again at the other test to check that the result was not just because they had learnt how the system operated. If a quickest time was recorded at a test then at least one attempt was made at the original test.

\section{RESULTS}

Typical results are shown in Fig. 5. The new systems described here were evaluated against an existing system [30,31] and to a driver driving a chair without any assistance. The vertical scale shows the average best time to complete various courses for drivers without using any sensors to assist (left in Fig. 5), using the recently published system [3,30] to compare (center) and improved system described in this paper (right). The horizontal scale lists the different testing routes.

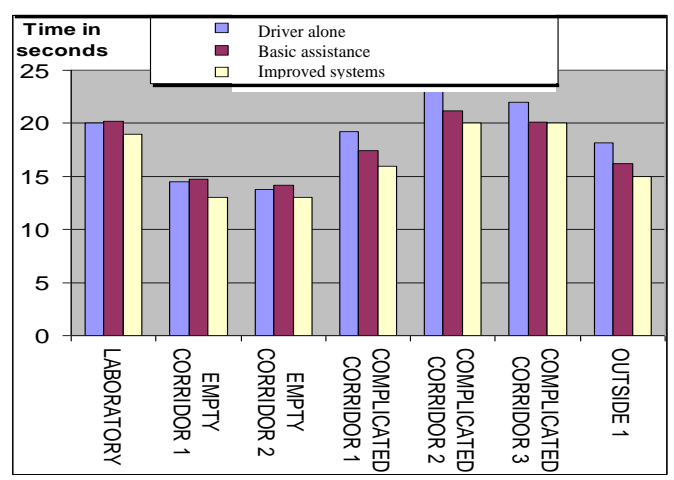

Figure 5. Results from tests.

On average, the system described in this paper performed faster. In simple environments (empty corridor and laboratory), drivers completed their routes faster without any assistance. In more complex environments (outside and complicated corridor), drivers completed routes faster with assistance. As environments became more complicated (or gaps became narrower) then human drivers found it more difficult to judge width of gaps or the angles required for them to safely pass through gaps. Human drivers sometimes had to slow their chair or stop and reverse to avoid collision. As environments became more complex, then drivers performed better with assistance. Items on walls sometimes slowed chairs as they were sensed but human drivers often ignored them. Different boundaries, slopes and surfaces tended to turn chairs and then sensors became more useful.
The new systems consistently and satisfactorily corrected wheelchair trajectories and out-performed recent systems.

\section{DISCUSSION AND CONCLUSIONS}

T-tests compared sample means. The mean and variation were calculated to give standard deviation. The separate individual sets of tests were not always statistically significant so care is needed before generalizing. A paired-samples statistical test could be used because pairs of results were recorded. Results were in two sets: result pairs with and without the sensors assisting. Pairing removed much of the random variability and results were statistically significant. The pairedsamples test showed that driving without sensors to assist and with sensors to assist were significantly different at $p<0.05$ ( $95 \%$ probability that the result would not occur by chance). The new system and methods described in this paper were significantly better than the recently published systems at $\mathrm{p}<$ 0.05 (95\% probability that this result would not occur by chance). On average, the new systems performed every test faster than the recently published system. Current new work is now investigating decision making [51-56].

\section{ACKNOWLEDGMENT}

Research in this paper was funded by EPSRC grant EP/S005927/1 and supported by The Chailey Heritage Foundation and University of Portsmouth.

\section{REFERENCES}

[1] D. Sanders and A. Hudson, "A specific blackboard expert system to simulate and automate the design of high recirculation airlift reactors" Math Comput Simulat 53 (1-2), pp. 41-65. 2000.

[2] D. Sanders, O. Okonor, M. Langner, et al, "Using a simple expert system to assist a powered wheelchair user". Adv. Intel. Syst. Comput., vol. 1037, Springer, pp. 662-379, 2019.

[3] D. Sanders "Comparing speed to complete progressively more difficult mobile robot paths between human tele-operators and humans with sensor-systems to assist" Assem Auto 29 (3), pp. 230-248, 2009.

[4] M.. Rahiman, Z. Zakaria and R. Rahim et al "Ultrasonic tomography imaging simulation of two-phase homogeneous flow" Sens. Rev 29 (3), pp. 266-276, 2009.

[5] D. Sanders "Controlling the direction of "walkie" type forklifts and pallet jacks on sloping ground" Assem Auto 28 (4), pp. 317-324, 2008.

[6] D. Sanders, G. Tewkesbury, H. Parchizadeh, et al, "Learning to drive with and without intelligent computer systems and sensors to assist". Adv. Intell. Syst. Comput. vol. 868, Springer, pp. 1171-1181, 2019.

[7] D. Sanders, A. Gegov, M. Haddad, F. Ikwan, D. Wiltshire, and Y. Tan, "A rule-based expert system to decide on direction and speed of a powered wheelchair". Adv. Intell. Syst. Comput. vol. 868, pp. 822-838, 2019.

[8] D. Sanders, A. Gegov, G. Tewkesbury, and R. Khusainov, "Sharing driving between a vehicle driver and a sensor system using trust-factors to set control gains". Adv. Intell. Syst. Comput. vol. 868, pp. 1182-1195, 2019.

[9] D.A. Sanders "Comparing ability to complete simple tele-operated rescue or maintenance mobile robot tasks with and without a sensor system" Sens. Rev, Vol 30, Issue 1, pp. 40-50, 2010.

[10] D.A. Sanders "Analysis of the effects of time delay on the tele-operation of a mobile robot in various modes of operation". Ind Rob Vol. 36 (6), pp. $570-584,2009$.

[11] I. Stott, and D. Sanders "New powered wheelchair systems for the rehabilitation of some severely disabled users" J Rehabil Res 23 (3), pp. 149-153, 2000. 
[12] D. Sanders, and I. Stott "A new prototype intelligent mobility system to assist powered wheelchair users" Ind Rob 26 (6), pp. 466-475, 1999.

[13] D.A. Sanders, and A. Baldwin, "X-by-wire technology" Total Vehicle Technology: Challenging current thinking, pp 3-12, 2001.

[14] D. Sanders, M. Langner, N. Bausch, et al, "Improving human-machine interaction for a powered wheelchair driver by using variable-switches and sensors that reduce wheelchair-veer". Adv. Intell. Syst. Comput. vol. 1038, pp. 1173-1191, 2019.

[15] D.A. Sanders, and G.E. Tewkesbury "A pointer device for TFT display screens that determines position by detecting colours on the display using a colour sensor and an ANN" Displays 30 (2), pp. 84-96, 2009.

[16] D.A. Sanders, S.D. Urwin-Wright, G.E. Tewkesbury et al, "Pointer device for thin-film transistor and cathode ray tube computer screens" Electronics Letters 41 (16), pp. 894-896. 2005.

[17] I. Stott, D. Sanders "The use of virtual reality to train powered wheelchair users and test new wheelchair systems" Int J Rehabil Res 23 (4), pp. 321326, 2000.

[18] M.J. Goodwin, D.A. Sanders. G.A. Poland et al "Navigational assistance for disabled wheelchair-users" Jrnl of Sys Arch 43 (1-5), pp. 73-79, 1997.

[19] D.A. Sanders, "The modification of pre-planned manipulator paths to improve the gross motions associated with the pick-and-place task Robotica 13 (Part 1), pp. 77-85, 1995.

[20] J. Larsson, M. Broxvall, and A. Saffiotti "Laser-based corridor detection for reactive Navigation”' Ind Rob: An int' jnl, 35 (1), pp. 69-79, 2008.

[21] S. Lee "Use of infrared light reflecting landmarks for localization" Ind Rob 36 (2), pp. 138-145, 2009.

[22] O Horn, and M. Kreutner "Smart wheelchair perception using odometry, ultrasound sensors, and camera" Robotica 27, pp. 303-310, 2009.

[23] V. Milanes, J.E. Naranjo and C. Gonzalez et al "Autonomous vehicle based in cooperative GPS and inertial systems” Robotica 26, pp. 627-633, 2008.

[24] D. Lim, S. Lee, and D. Cho "Design of an assisted GPS receiver and its performance analysis", IEEE Symp on Circ' \& Syst, pp. 1742-1745, 2007.

[25] R. Bloss "Vision and robotics team up at the 2007 Show". Ind Rob: An int' jnl 35 (1), pp. 19-26, 2008.

[26] D. Sanders, Y. Tan, I. Rogers and G. Tewkesbury "An expert system for automatic design-for-assembly" Assem Auto, vol. 29 (4), pp. 378-388, 2009.

[27] D. Sanders "Environmental sensors and networks of sensors" Sens. Rev. 28 (4), pp. 273-274, 2008.

[28] D. Hopper "The long perspective for robotic vision" Assembly Automation 29 (2), pp 122-126, 2009.

[29] D. Sanders, G. Lambert, J. Graham-Jones, et al. “A robotic welding system using image processing techniques and a CAD model to provide information to a multi-intelligent decision module" Assem Auto, Vol. 30 (4), pp. $323-332,2010$.

[30] D. Sanders, J. Graham-Jones, A. Gegov, "Improving ability of teleoperators to complete progressively more difficult mobile robot paths using simple expert systems and ultrasonic sensors", Ind Rob - An Int Jrnl Vol 37 (5), pp. 431-440, 2010.

[31] D. Sanders, G. Lambert and L. Pevy "Pre-locating corners in images in order to improve the extraction of Fourier descriptors and subsequent recognition of shipbuilding parts" IMechE Part B, vol 223 (9), pp. 1217 $1223,2009$.

[32] D. Sanders "Progress in machine intelligence" Ind Rob vol 35 (6), pp. 485-487, 2008.

[33] [33] D. Sanders "Recognizing shipbuilding parts using artificial neural networks and Fourier descriptors" IMechE Part B, vol 223 (3), pp. 337342, 2009.

[34] D. Sanders and I. Stott "Analysis of failure rates with a tele-operated mobile robot between a human tele-operator and a human with a sensor system to assist" Robotica Vol 30 (6), pp. 973-988, 2012.

[35] D. Sanders, M. Langner and G. Tewkesbury "Improving wheelchairdriving using a sensor system to control wheelchair-veer and variable- switches as an alternative to digital-switches or joysticks" Ind Rob: An International Journal, Vol. 37 (2), pp. 157 - 167, 2010.

[36] O. Khatib "Real-time obstacle avoidance for manipulators and mobile robots", Proc of IEEE Int. Conf. on Robotics and Automation, Vol. 5 No.1, pp.90-98, 1986.

[37] A. Hudson, D. Sanders and H. Golding et al "Aspects of an expert design system for the wastewater treatment industry" J Syst Architect, 43 (1-5), pp. 59-65, 1997.

[38] D. Sanders "Introducing AI into MEMS can lead us to brain-computer interfaces and super-human intelligence" (invited viewpoint review paper) Assembly Autom Vol. 29 (4), pp. 309-312, 2009.

[39] D. Sanders "Ambient-intelligence, rapid-prototyping and where real people might fit into factories of the future" Assembly Autom 29 (3), pp. 205-208, 2009.

[40] D. Sanders, B. Haynes and G. Tewkesbury et al, "The addition of neural networks to the inner feedback path in order to improve on the use of pretrained feed forward estimators" Math Comput Simulat 41 (5-6), pp. 461 472, 1996.

[41] D. Sanders, "Perception in robotics" Ind Rob vol 26 (2), pp. 90-92, 1999.

[42] D. Sanders, "System Specification 2". Microproc Microprog 38 (1-5), pp. 833-833, 1993.

[43] D. Sanders, P. Harris and E. Mazharsolook "Image modellling in realtime using spheres and simple polyhedra "4th Int Conf on Image processing and its applications 354, pp. 433-436, 1992.

[44] D. Sanders, A. Hudson and G. Tewkesbury " Automating the design of high-recirculation airlift reactors using a blackboard framework" Expert Syst Appl 18 (3), pp. 231-245, 2000.

[45] D. Sanders "Real time geometric modelling using models in an actuator space and cartesain space" Jrnl of robotic systems 12 (1), pp. 19-28, 1995.

[46] F. Fahimi, C. Nataraj and H. Ashrafiuon "Real-time obstacle avoidance for multiple mobile robots" Robotica 27, pp. 189-198, 2009.

[47] G. Tewkesbury and D. Sanders "The automatic programming of production machinery for de-flashing plastic parts" Advances in manufacturing technology VIII, pp. 279-283, 1994.

[48] G. Tewkesbury and D. Sanders "The use of distributed intelligence within advanced production machinery for design applications" Total vehicle technology: Challenging current thinking, pp 255-262, 2001.

[49] D. Sanders, D. Robinson, M. Hassan Sayed, M. Haddad, A. Gegov, Aand N. Ahmed, "Making decisions about saving energy in compressed air systems using Ambient Intelligence and Artificial Intelligence". Adv. Intell. Syst. Comput. vol. 869, Springer, pp. 1229-1236, 2019.

[50] D. Sanders, Q. Wang, N. Bausch, et al, "A method to produce minimal real time geometric representations of moving obstacles". Adv. Intell. Syst. Comput. vol. 868, pp. 881-892, 2019.

[51] M. Haddad, D. Sanders, A. Gegov, et al, "Combining multiple criteria decision making with vector manipulation to decide on the direction for a powered wheelchair". Adv. Intell. Syst. Comput. vol. 1037, Springer, pp. 680-693, 2019.

[52] M. Haddad, D. Sanders, and N. Bausch, 'Selecting a robust decision making method to evaluate employee performance', International Journal of Management and Decision Making, vol. 18, no. 4, pp. 333-351. 2019.

[53] M. Haddad, D. Sanders, and G. Tewkesbury, 'Selecting a discrete Multiple Criteria Decision Making method to decide on a corporate relocation', Archives of Business Research, vol. 7, no. 5, pp. 48-67. 2019.

[54] M. Haddad, and D. Sanders"Selecting a best compromise direction for a powered wheelchair using PROMETHEE", IEEE Trans Neural Syst Rehabil Eng, vol. 27, no. 2, pp. 228-235. 2019.

[55] M. Haddad, D. Sanders, G. Tewkesbury, et al, "Initial results from using Preference Ranking Organization METHods for Enrichment of Evaluations to help steer a powered wheelchair". Adv. Intell. Syst. Comput. vol. 1037, pp. 648-661, 2019.

[56] M. Haddad, D. Sanders, N. Bausch, et al, "Learning to make intelligent decisions using an Expert System for the intelligent selection of either PROMETHEE II or the Analytical Hierarchy Process". Adv. Intell. Syst. Comput. vol. 868, pp. 1303-1316, 2019 